BMC

Genomics

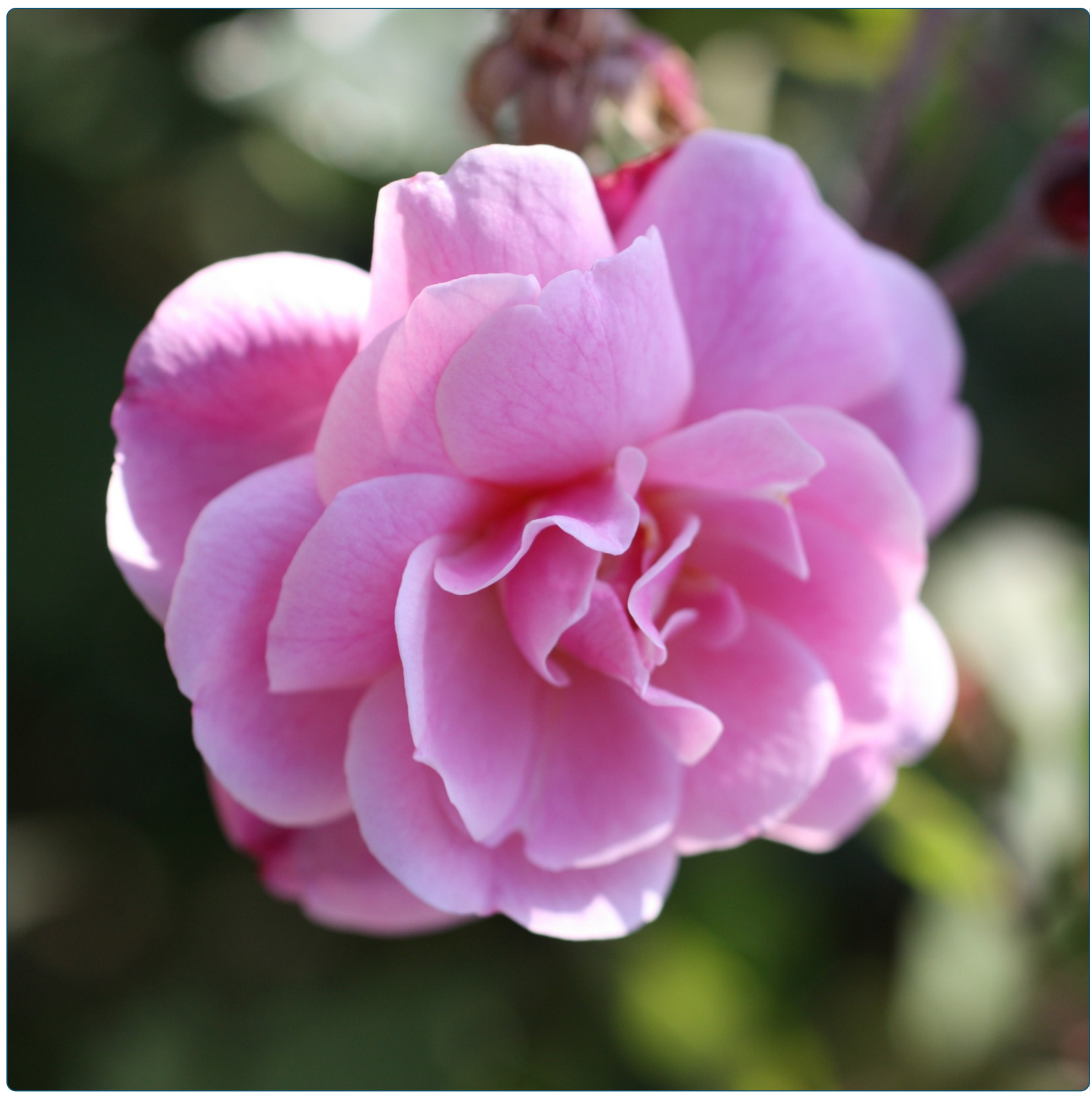

Transcriptome database resource and gene expression atlas for the rose

Dubois et al.

() Biomed Central 


\title{
Transcriptome database resource and gene expression atlas for the rose
}

\author{
Annick Dubois $^{1 \dagger}$, Sebastien Carrere ${ }^{2,3 \dagger}$, Olivier Raymond ${ }^{1}$, Benjamin Pouvreau', Ludovic Cottret ${ }^{2,3}$, \\ Aymeric Roccia ${ }^{1,4}$, Jean-Paul Onesto ${ }^{5}$, Soulaiman Sakr ${ }^{6}$, Rossitza Atanassova ${ }^{7}$, Sylvie Baudino ${ }^{4}$, Fabrice Foucher ${ }^{6}$, \\ Manuel Le Bris ${ }^{8}$, Jérôme Gouzy ${ }^{2,3}$ and Mohammed Bendahmane ${ }^{1 *}$
}

\begin{abstract}
Background: For centuries roses have been selected based on a number of traits. Little information exists on the genetic and molecular basis that contributes to these traits, mainly because information on expressed genes for this economically important ornamental plant is scarce.

Results: Here, we used a combination of Illumina and 454 sequencing technologies to generate information on Rosa sp. transcripts using RNA from various tissues and in response to biotic and abiotic stresses. A total of 80714 transcript clusters were identified and 76611 peptides have been predicted among which 20997 have been clustered into 13900 protein families. BLASTp hits in closely related Rosaceae species revealed that about half of the predicted peptides in the strawberry and peach genomes have orthologs in Rosa dataset. Digital expression was obtained using RNA samples from organs at different development stages and under different stress conditions. qPCR validated the digital expression data for a selection of 23 genes with high or low expression levels. Comparative gene expression analyses between the different tissues and organs allowed the identification of clusters that are highly enriched in given tissues or under particular conditions, demonstrating the usefulness of the digital gene expression analysis. A web interface ROSAseq was created that allows data interrogation by BLAST, subsequent analysis of DNA clusters and access to thorough transcript annotation including best BLAST matches on Fragaria vesca, Prunus persica and Arabidopsis. The rose peptides dataset was used to create the ROSAcyc resource pathway database that allows access to the putative genes and enzymatic pathways.
\end{abstract}

Conclusions: The study provides useful information on Rosa expressed genes, with thorough annotation and an overview of expression patterns for transcripts with good accuracy.

Keywords: Rose, Transcriptome, Gene expression atlas

\section{Background}

Cultivated roses have a very ancient history and artificial crossing led to what are today perceived as the "modern rose cultivars". Rose (genus Rosa) belongs to the large family of the Rosaceae (e.g. apple, strawberry or peach). Roses are of a high symbolic value and a great cultural importance in different societies. They are widely used as garden ornamental plants and as cut flowers. Earlier

\footnotetext{
* Correspondence: mohammed.bendahmane@ens-lyon.fr

'Equal contributors

DDeceased

'Reproduction et Développement des Plantes UMR INRA-CNRS- Université Lyon 1-ENSL, Ecole Normale Supérieure, 46 allée d'Italie, Lyon Cedex 07 69364, France

Full list of author information is available at the end of the article
}

domestication of roses involved selection for a number of traits, mainly involving floral quality, such as recurrent flowering, double flowers, petal color and fragrance. Very little information is available on the molecular mechanisms that control these traits. This dearth of information limits the scope of rational selection for improvement of ornamental plants. Rose breeding practices often involve introgression of desirable traits (mainly floral) from nonelite or wild varieties with varying ploidy levels into tetraploid elite cultivars. Selection pressure has also led to the loss of important characters such as tolerance to biotic and/or abiotic stresses. In many crop species, molecular markers allow breeders to rapidly screen a large number of lines for markers associated with traits of interest,

\section{Biomed Central}


allowing the subsequent selection of relevant molecular markers and thus specific introgression of single genomic loci. However, in roses the lack of knowledge of the genetic basis upon which modern rose cultivars are established hampers molecular marker assisted selection.

The enormous progress that has been made towards understanding various aspects of plant development and resistance to biotic and abiotic stresses, as well as defining the molecular and genetic pathways associated with these processes, has mainly involved annual model species such as Arabidopsis thaliana, tobacco, rice or maize. Several traits such as recurrent blooming, scent production and the production of double flowers, cannot be studied using these model species, or at least only in a limited manner. The rose represents an ideal ornamental model species to address some of these characters.

During the past few years, EST sequencing using cDNA libraries has been used to identify genes expressed in Rosa $s p$ [1-3]. However, these rose ESTs remained limited to genes expressed during floral development and to date sequence information for only about 5000 genes exists in the databases [4]. Despite their relatively limited number, the available ESTs for the rose have been valuable for the identification of several novel genes associated with flower characters such as the scent related germacrene D synthase, O-methyltransferases and alcohol acetyltransferase genes [3,5-10]. In recent years, Rosa chinensis cv. Old Blush was chosen as a model to develop tools for genomics and genetic transformation $[4,11]$. This diploid and recurrent flowering rose is a common ancestor of many commercial modern roses and has contributed to the recurrent flowering and the tea scent traits [12].

Here we used a combination of 454 and Illumina sequencing technologies to establish an EST database containing information on rose sequences expressed in a wide range of $R$. chinensis Old Blush tissues as well as during biotic and abiotic stresses. An in silico profile of gene expression and a reconstruction of rose metabolic pathways are presented and have been made accessible through web interface. Real time quantitative RT-PCR (qPCR) analyses of selected genes whose expression is specific to different organs, stress conditions and/or development stages confirmed the in silico data. Similarly, rose genes associated with flower development, stamen development and fragrance show consistent in silico expression patterns.

\section{Results and discussion}

\section{RNA sampling, experimental design}

With the objective of increasing the available coding sequences for the rose, RNA samples were prepared from various organs of $R$. chinensis cv. Old Blush plants, grown under controlled greenhouse conditions, and then used to generate unidirectional cDNA libraries. To get a wide representation of genes expressed in Rosa $s p, 13$ RNA samples representing all rose plant organs and tissues were generated. Samples include vegetative and floral meristems, buds at different steps of bursting, floral organs at different developmental stages, young (white) developing roots, rose hips (cynorhodon) at early developmental stages, untreated young leaves and leaves that were subjected to biotic or abiotic stresses (Figure 1). Leaves were water stressed or infected with Botrytis cinerea LR18, a necrotrophic fungal pathogen known to cause severe symptoms such as grey mold in many soft fruits and ornamentals including rose, gerbera and chrysanthemum [13]. Infection by $B$. cinerea reduces the postharvest quality of rose flowers leading to substantial economic loss by growers and wholesalers [14].

\section{EST sequencing and clustering, sequence annotation and database creation}

To obtain a reference set of transcripts, a normalized cDNA library from the above 13 different rose tissues and conditions was sequenced using Roche GS-FLX 454 Titanium technology. About 1,043,708 raw reads with an average length of 350 nucleotides corresponding to a total of $288 \mathrm{Mb}$ were obtained (Table 1). In parallel, the 13 different non-normalized cDNA libraries were individually barcoded and sequenced using the Illumina technology. About 9332571 reads with a minimum length of 32 nucleotides corresponding to about $300 \mathrm{MB}$ were obtained and assembled using edena ( - overlapCutoff $=22$; minContigSize $=80$ ) [15].

Clustering was performed using a modified version of TGICL optimized to accommodate very large datasets [16]. The input sequences were both trimmed 454 reads and 20554 contigs generated by edena using the short Illumina reads. A total of 80714 rose EST clusters longer than 100 nucleotides and based on more than 2 sequence fragments were assembled. Each fragment originated either from a 454 read or from an edena contig. These Rosa sp. EST sequences are available at the ROSAseq web interface database, http://iant.toulouse.inra.fr/R. chinensis. An additional 1248 clusters had significant matches in the Botrytis cinerea genome and are available as a separate set available as a tabulated file (under " $B$. cinerea alignments") on the ROSAseq web interface database.

11307 rose cDNA clusters contained more than 15 reads and only 32 clusters contained over 200 reads, amongst which 3 had more than 300 reads (with a maximum of 447 reads for the most represented cluster). These figures indicate that normalization of the reference library from pooled tissue was particularly efficient (Additional file 1: Figure S1). The set of clusters that had more than 200 reads contained genes known to be highly expressed, such as genes coding for proteinase 

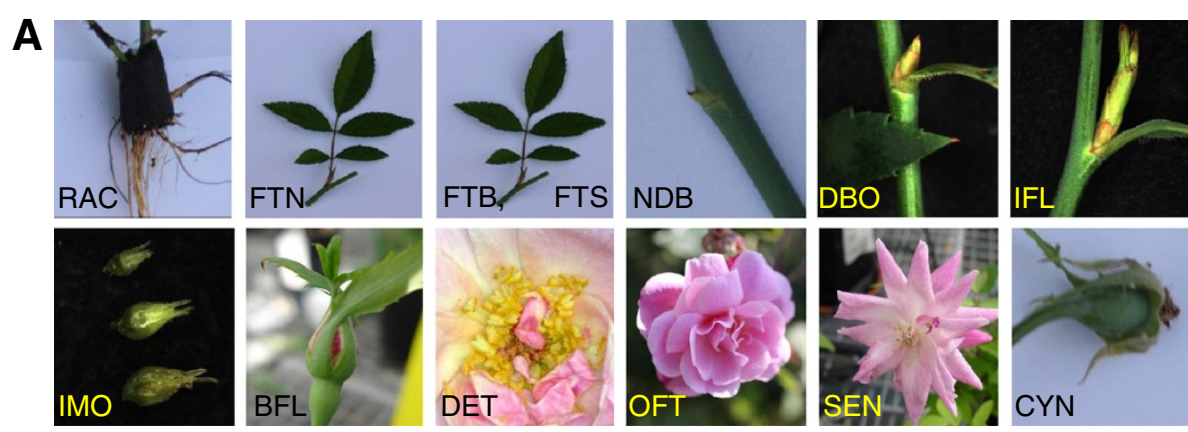

.

\section{B}
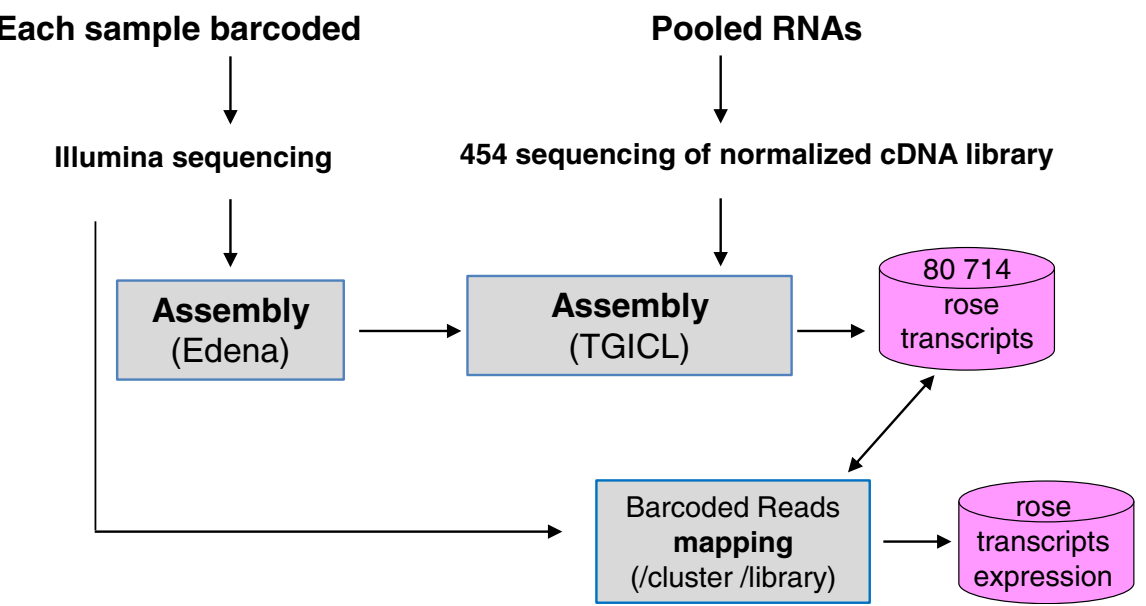

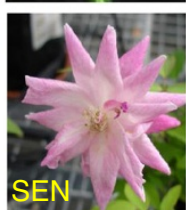

\section{Pooled RNAs}
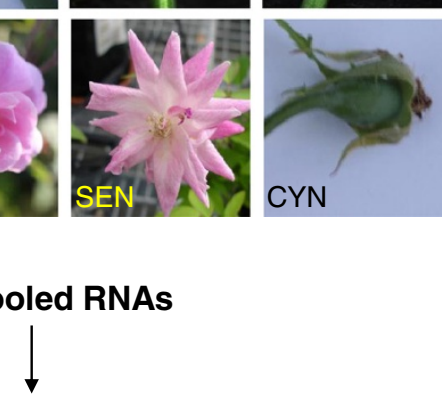

Figure 1 Samples description and experimental design for next-generation sequencing. A) 13 different tissues and conditions used for RNA purification. RAC: White young roots; FTN : Young leaves and stems; FTB : Leaves infected with Botrytis cinerea LR18; FTS: Leaves from water stressed plants; NDB: Dormant axillary buds (vegetative meristem); DBO: Active axillary buds (vegetative meristem); IFL: Floral bud at floral meristem transition; IMO: Floral meristem and early floral organs (sepal, petal, stamens and carpels) development; BFL: closed flower; DET: Stamens at microsporogenesis and microgametogenesis stages; OFT: open flower; SEN: senescent flower; CYN: rose hip from pollination up to early pigmentation. B, Sequencing and assembly strategy. Illumina reads were assembled using edena and combined with the trimmed 454 reads using TGICL to generate the final clusters assembly.

Table 1 Raw data from Illumina and 454 sequencing

\begin{tabular}{llll}
\hline & Sample & Number of reads & Number of bases \\
\hline Illumina raw data & NDB & 620667 & 19861344 \\
& DBO & 407020 & 13024640 \\
& IFL & 746757 & 23896224 \\
& IMO & 862350 & 27595200 \\
& BFL & 913850 & 29243200 \\
& OFT & 1200674 & 38421568 \\
& DET & 670449 & 21454368 \\
& SEN & 726993 & 23263776 \\
& CYN & 577706 & 18486592 \\
& FTN & 485234 & 15527488 \\
& FTS & 823657 & 26357024 \\
& FTB & 451500 & 14448000 \\
& RAC & 845714 & 27062848 \\
& Total & 9332571 & 298642272 \\
\hline
\end{tabular}

inhibitors, histones, and ribosomal proteins, but also genes with more specific expression patterns such as the floral organ identity MADS-box transcription factor APETALA3, and a putative terpenoid synthase coding gene whose expression is specific to mature floral tissue [17].

The clusters' best BLASTN hits in closely related Rosaceae species with sequenced genomes (strawberry and peach, e-value1e-6) revealed that 44656 clusters had a BLASTN hit on 14252 Fragaria vesca transcripts with a mean nucleotide identity of $90,88 \%$, and 36455 clusters had hits on 13033 Prunus persica genes with an average nucleotide identity of $85,01 \%$. Peach, strawberry and rose have relatively small genome sizes of about $230 \mathrm{Mb}, 240$ $\mathrm{Mb}$ and $560 \mathrm{Mb}$ respectively, and exhibit high synteny $[18,19]$. In the strawberry and peach genomes there are 34809 and 27852 predicted transcripts respectively, not all of them being supported by transcriptome mapping ([20]; http://www.rosaceae.org/peach/genome). Overall, about $53 \%$ and $44 \%$ of the predicted transcripts in the strawberry and peach, respectively, are represented in 
our Rosa sp. database. BLASTN (e-value 1e-6) between strawberry and peach transcripts showed that 25543 strawberry transcripts have hits on 16777 peach transcripts (65\% of total peach transcripts) and 26522 peach transcripts have blast hits on 17625 strawberry transcripts (66\% of total strawberry transcripts). Therefore, the observed slightly lower percentage of rose transcripts with hits in strawberry or peach transcripts can be due to the fact that some tissues or developmental stages are missing in our sampling combined with non exhaustive sequencing depth.

To have predictive peptide information, the 80714 clusters were analyzed with the FrameDP [21]. 76611 sequences were predicted to code for putative proteins which were annotated based on an automatic InterproScan analysis [22]. The OrthoMCL tool was used to generate families of proteins where each family consists of orthologs or "recent" paralogs from at least two species with a whole genome sequence [23]. Rose predicted peptides were compared to the proteomes from $F$. vesca, $P$. persica and $A$. thaliana. This method uses an allagainst-all BLAST search (including within-genus and between genus BLAST) of each genus proteome, followed by a Markov cluster algorithm. The analysis is based on a BLASTp with stringent parameters, followed by a computation excluding sequences with Percent Match Cutoff lower than $80 \%$. OrthoMCL analysis clustered 20997 putative rose peptides into 13900 protein families. 8769 OrthoMCL families corresponded to unique Rosa sp. genes, 4074 families corresponded to two genes and 1057 corresponded to more than two genes. The orthoMCL families that corresponded to at least two genes represent either proteins coded by different alleles or peptides from the same protein but with no overlapping amino acid sequence. Alternatively, the multiple gene families may correspond to genes subject to recent duplication events.

The second level of OrthoMCL analysis allowed normalized inter-species comparisons (Figure 2). Common and specific OrthoMCL families (including paralogs and orthologs) were identified in the different species. The rose protein dataset contains 9518, 9302 and 8179 common families with the $F$. vesca, $P$. persica and $A$. thaliana proteomes, respectively. OrthoMCL analysis allowed the identification of 3561 gene families that appeared unique to the Rosa genus when compared to $F$. vesca, $P$. persica and $A$. thaliana. However, this number of gene families unique to Rosa $s p$ is likely to be an overestimate since certain families may not exhibit sufficient overlap with their hit from another species. We identified 2558 peptides in the Rosa dataset that share a unique ortholog in the four analyzed species, Rosa, Prunus, Fragaria and Arabidopsis. Access to the protein sequences in fasta format for each OrthoMCL cluster is

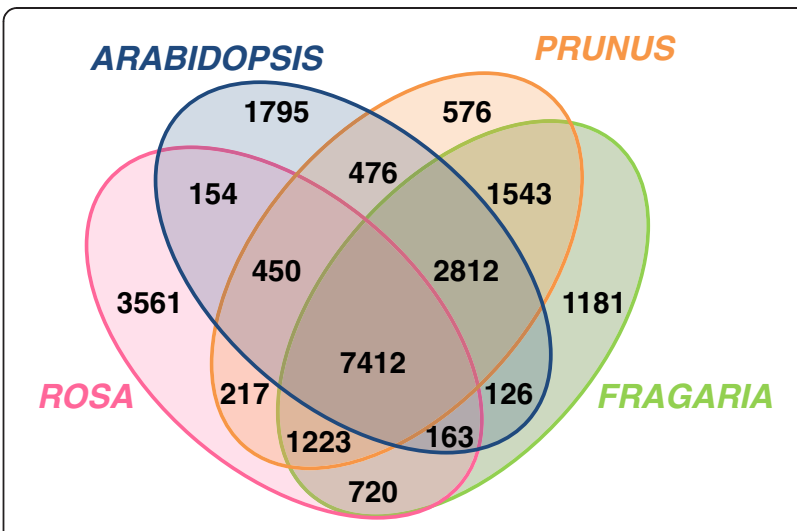

Figure 2 Gene families shared between Rosa chinensis, Fragaria vesca, Prunus persica and Arabidopsis thaliana. OrthoMCL program was used to identify gene families shared between the four species using the following BLASTp parameters : P-value Cut-off 1e-05; Percent Identity Cut-off 0 [-F F] (i.e. low complexity filter inactivated).

To increase confidence, a percent Match Cut-off 80 (i.e. Query and Match had to overlap on more than $80 \%$ of the query and match sequence length) filter was performed on the BLAST results.

possible through the web portal http://iant.toulouse.inra. fr/R.chinensis.

\section{Gene representation in different putative pathways}

Pathway Tool [24] was applied to generate a dedicated resource using the rose peptide dataset. The putative pathways identified using semi-automated tools are available at http://pathway-tools.toulouse.inra.fr/ROSACYC under ROSAcyc. The majority of the previously reported pathways in plants are present in the ROSAcyc database and can be viewed through the web portal. For example, analyses of the secondary metabolism pathways showed that the carotenoid biosynthesis superpathway is well supported in the ROSAcyc database by numerous putative peptides (http://pathway-tools.toulouse.inra.fr/ ROSACYC/new-image? type $=$ PATHWAY\&object $=$ CAROTENOID-PWY). The database provides information on peptides that were automatically attributed to a given metabolic pathway. Such information can be used as a basis for further data mining, such as searches of gene expression patterns.

\section{In silico expression patterns of Rosa sp genes}

A molecular tagging approach coupled to Illumina sequencing was used to construct an "in silico gene expression atlas" of different rose tissues and stress conditions. The 13 different non-normalized cDNA libraries representing various rose tissues and conditions (Figure 1) were individually barcoded and sequenced using the Illumina technology (Table 1). The 9332571 short reads were mapped using glint software (Faraut $\mathrm{T}$. and Courcelle E.; http://lipm-bioinfo.toulouse.inra.fr/ download/glint/, unpublished) onto the EST clusters 
generated from the 454 sequencing data and counted per cluster and per library. For each cluster, total short reads counted per library are available, providing clues towards an expression pattern for the corresponding gene. RPKM normalization [25] was also performed. Raw and normalized counts are available for each cluster in the database.

These data were first validated through a qPCR approach. Twenty-three genes whose expression was previously reported to correlate with certain physiological characters, such as color, scent biosynthesis, pollen or egg cell ploidy level, as well as developmental characters (branching, recurrent flowering, form and number of organs especially petals) in Rosa $s p$ and/or in other species such as A. thaliana, were selected for qPCR expression profiling (Figure 3; Additional file 2: Figure S2). The correlation between RNAseq/in silico data and qPCR data was assessed by calculating the Pearson's product moment correlation coefficient (Additional file 3: Table S1). The statistical significance of each Pearson's correlation coefficient was assessed using the cor.test routine in R. For most analyzed genes a high correlation coefficient was observed (mean of 0.81 ) and only a few genes showed low correlation between qPCR and in silico data (Additional file 3: Table S1). These results suggest that our in silico data is accurate in the different tissues and experimental conditions.

In silico expression can be obtained for each of the 80714 clusters, through the web portal ROSAseq. To address whether this atlas of gene expression allows data mining to help initiating studies of specific functions in the rose, the following three important developmental processes related to flower development and scent were analyzed.

Rose genes involved in flower initiation and development We addressed the expression profiles for transcripts previously shown to exhibit flower specific expression patterns in Rosa as well as for putative orthologs of well-described Arabidopsis floral genes (Additional file 4: Table S2;
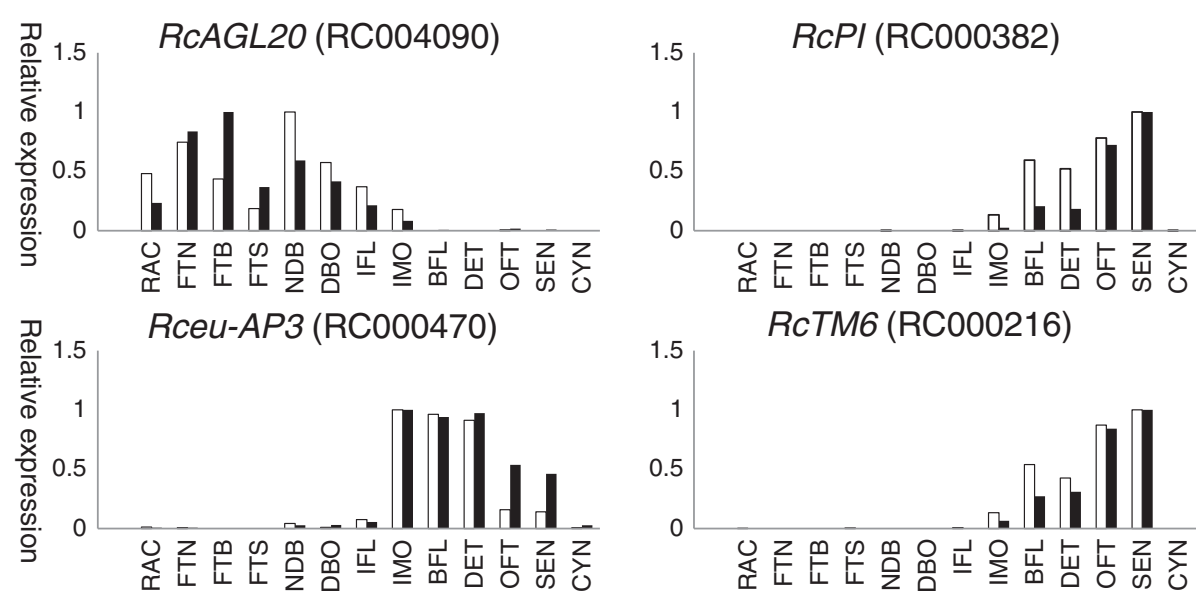

RcTM6 (RC000216)
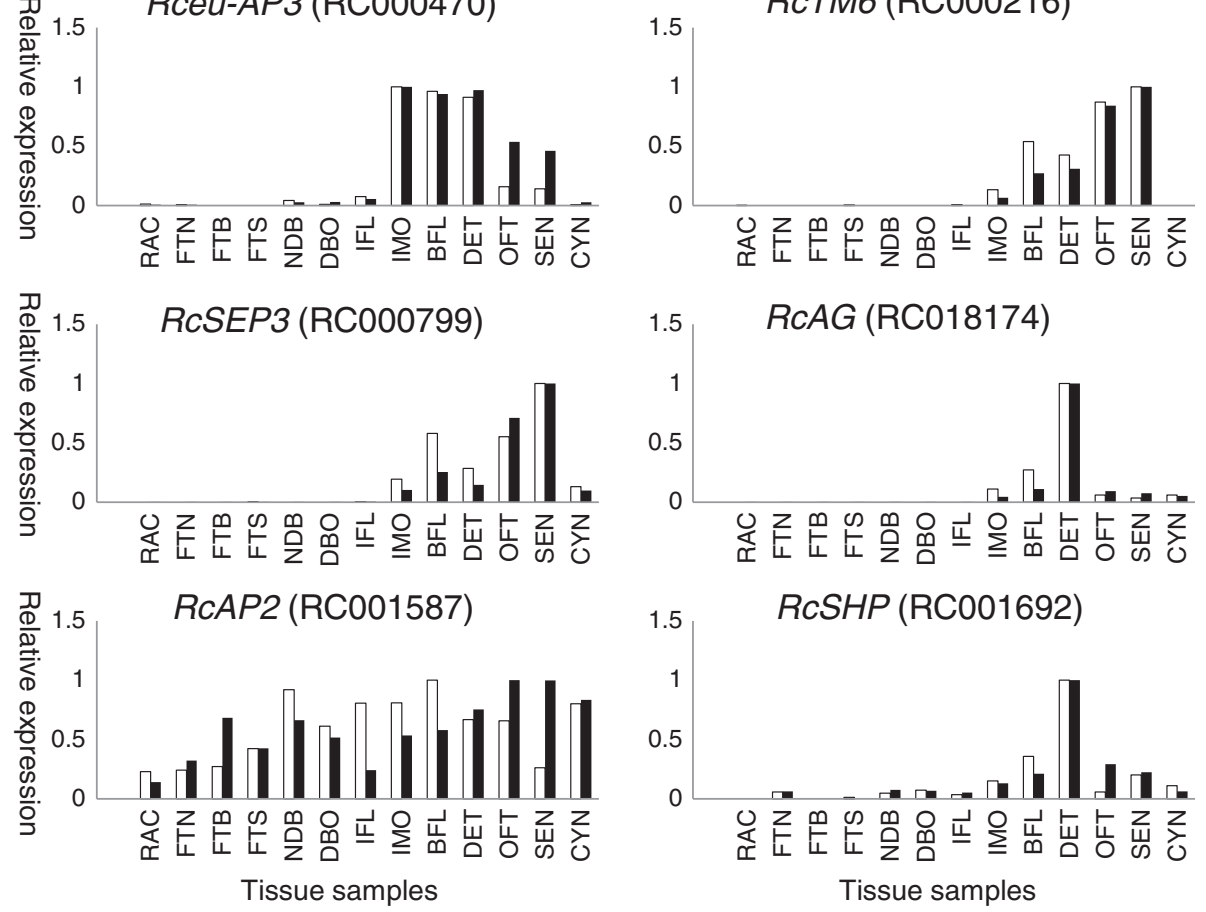

Figure 3 Expression analyses by real time quantitative RT-PCR (qPCR) of eight transcripts selected in silico. Relative qPCR and RPKM values were scaled with maximum expression value set to 1. White histograms: relative RPKM counts; black histograms: qPCR relative expression. 
Figure 4A). Because our dataset is fragmented due to the nature of the next generation sequencing techniques employed [26], for each Arabidopsis gene, more than one cluster was identified in the ROSAseq dataset. Interestingly, the clusters corresponding to the same gene showed similar expression patterns, thus providing another argument of the reliability of the in silico expression data.

Transcripts encoding the putative floral integrator RcSOC1 (SUPPRESSOR OF CONSTANS 1, represented by cluster RC019456) accumulate during the vegetative phase and the floral initiation stage. No accumulation of this putative $R c S O C 1$ transcript was detected during later stages of floral development. This pattern of RcSOC1 expression has already been observed in Rosa [27], suggesting a role of SOC1 homologs during the vegetative phase and the floral transition. During the floral initiation process, transcripts of the ortholog of APETALA1 (RcAP1) accumulate progressively with a maximum expression during early floral organogenesis (IMO), in agreement with previously reported data in Rosa sp. [27].

Transcripts corresponding to putative MADS box transcription factors involved in floral organ identity specification were represented in the ROSAseq dataset. Transcripts of the putative B class genes RcTM6-clade (RCTM6), RcAPETALA3 (RcAP3) and RcPISTILLATA $(R c P I)$ were detected at high levels in all floral samples, consistently with previously reported data [28]. Although expressed at lower levels, transcripts encoding putative orthologs of AGAMOUS (AG), SEEDSTICK and SHATTERPROOF (SHP) were detected in floral samples and in the cynorhodon (rose hip). This result is consistent with previously reported data on the rose RhAGAMOUS (RhAG) orthologs $[29,30]$. Interestingly, the clusters corresponding to the putative SHP1 did not show any particular enrichment in the hip library, but exhibited an expression pattern similar to that of $R h A G$ in developing stamens. This pattern resembles the previously reported expression profile of C-function genes in Petunia and Antirhinnum, suggesting a shared C-function between $A G$ and SHP orthologs [31]. As expected, putative homologs of the key floral developmental regulator genes SEPALLATA1 (SEP1) and $S E P 3$ were expressed in rose floral tissues. Interestingly, RcSEP1 and RCSEP3 were also expressed in the cynorhodon. In agreement with this observation, the strawberry FvSEP1/2 subfamily genes have been shown to be involved in post-fecondation receptacle tissues development and ripening [32] and LeSEP3-like genes are necessary for fruit ripening in tomato [33].

\section{Genes expressed during stamen development}

In Rosa sp no information is available on the regulatory cascades of genes regulating anther development and

\section{A}

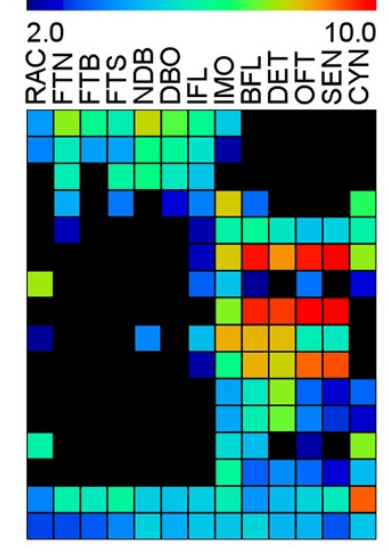

C

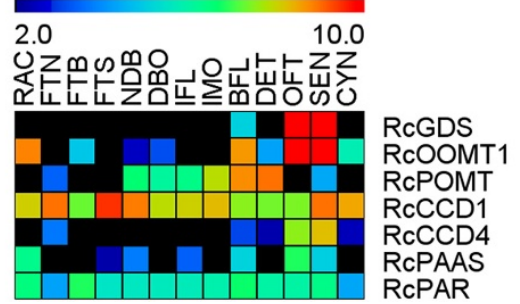

B

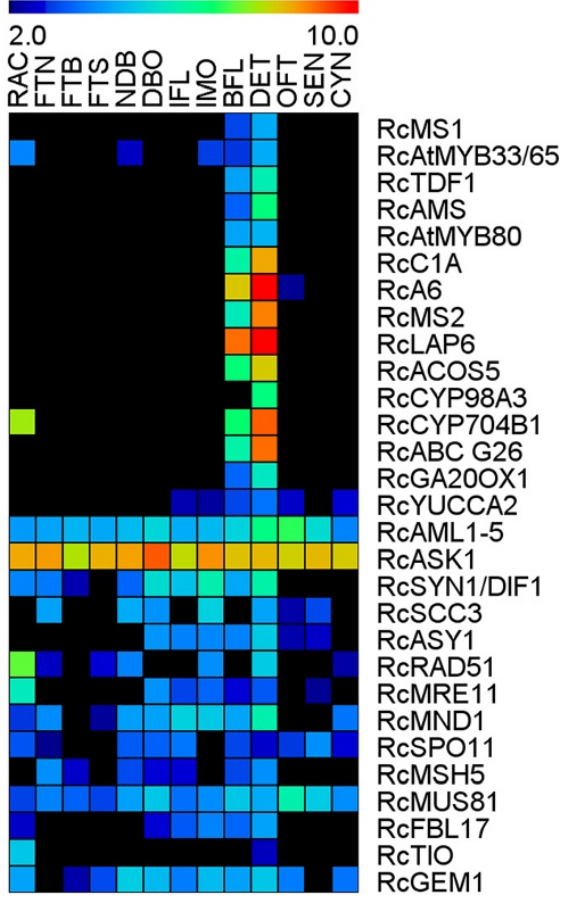

Figure 4 Expression profiles of selected transcripts putatively associated with flower development and scent. Floral development (A), stamen formation and meiosis (B) and scent biosynthesis (C) associated transcripts. Heatmap was generated using RPKM data (In2 scale) using the MeV analysis tool [64]. 
meiosis. We performed a BLAST search using genes from Arabidopsis known to be involved in both stamen development and male gametogenesis [34-40]. Candidate clusters were readily identified in the ROSAseq dataset (Additional file 4: Table S2), and their in silico expression profile was analyzed further (Figure 4B). Orthologs of genes involved in stamen development and microsporogenesis exhibited an expression in rose stamens (DET) and flower bud (BFL) samples, and were absent from other tissues. This is the case for MALE STERILITY1 (MS1), ABORTED MICROSPORE (AMS) and DEFECTIVE IN TAPETAL DEVELOPMENT AND FUNCTION1 (TDF1), three genes known to be involved in tapetum development and/or microsporogenesis in Arabidopsis [41-44].

Orthologs of gene previously shown to be involved in early meiosis events up to microspore release in Arabidopsis are also represented in the rose stamens DET sample (Figure 4B). The $A 6$ ortholog showed specific expression in the rose stamens [45], while homologs of AML1-5 and ASK1; [46,47] did not exhibit enrichment in the DET sample, thus in agreement with data reporting $A M L$ and ASK1 genes expression in both vegetative and reproductive tissues in Arabidopsis [46,48].

Similar to Arabidopsis, orthologs of genes involved in pollen wall formation (ACOS5, CYP98A3; $[49,50])$ or in sperm cell specification and division genes (FBL17) [51] exhibited an expression enriched in the rose stamen DET sample.

Taken together, these analyses demonstrate that this rose gene expression atlas is a reliable source for candidate genes associated with male reproductive processes.

\section{Scent Biosynthesis genes representation in the ROSAseq database}

We searched the database for genes previously shown to be involved in rose scent biosynthesis (Figure 4C; Additional file 4: Table S2). Transcripts coding for the putative germacrene-D synthase (RcGDS) accumulated at high levels during flower opening and senescence (OFT and SEN samples) while low expression was observed in flower bud samples (BFL). It has been reported that the germacrene-D biosynthesis occurs during anthesis and at the onset of senescence [3]. Therefore, our in silico data shows that the accumulation of GDS transcripts correlates with germacrene-D biosynthesis.

Transcripts coding for two enzymes involved in the biosynthesis of the 1,3,5-trimethoxybenzene (TMB) are represented in the ROSAseq dataset. The transcript coding for phloroglucinol O-methyltransferase (POMT), known to catalyze the methylation of phloroglucinol to 3,5 dihydroxyanisole [52] is highly expressed in flower buds (BFL) and stamens (DET). The 3,5 dihydroxyanisole is a precursor for TMB $[6,7,52]$. The transcripts corresponding to the orcinol-O-methyl transferases (RcOOMT1 and RcOOMT2), known to act downstream in TMB and 3,5 dimethoxytoluene (DMT) biosynthesis, exhibited an expression during anthesis and senescence (OFT and SEN) (Figure 4C; Additional file 2: Figure S2), thus in agreement with previously reported data [6]. RcOOMT1 and RcOOMT2 originated from a recent gene duplication and exhibit high nucleotide identity and thus their expression cannot be discriminated [6,8].

The carotenoid cleavage oxygenases CCD1 and CCD4 have been reported to exhibit high expression levels in flowers and to be involved in the biosynthesis of terpenes, such as beta-ionone[53,54]. Similarly, our in silico data shows high accumulation of the putative RcCCD1 transcripts in rose flowers (Figure $4 \mathrm{C}$ ), but also in vegetative organs, in agreement with previously reported data [53]. Our in silico data shows that the putative RcCCD4 transcripts accumulate to high levels in the flower and more specifically during flower opening and senescence (OFT and SEN), thus consistent with previously reported data [54].

2-phenylethyl alcohol is another organic volatile compound responsible for typical rose scent. Its synthesis occurs via two steps in Rosa sp. The first step is catalyzed by the phenylacetaldehyde synthase (PAAS), converting phenylalanine to phenylacetaldehyde [55]. Phenyacetaldehyde reductase (PAR) catalyzes the second step, reducing the phenylacetaldehyde to 2-phenylethyl alcohol [56]. In silico data showed that RcPAAS was expressed at low levels during late floral development. This result is not surprising, as $R$. chinensis Old Blush flowers do not produce phenylethanol [6]. According to our in silico data $R C P A R$ expression was not restricted to flowers, thus in agreement with previous reported data [57].

Taken together these three examples show that the rose in silico expression atlas appears accurate and provides a valuable resource for $a b$ initio gene expression analysis. For each cluster, exhaustive annotation has been performed and can be obtained through the web portal. This annotation data, combined with expression data for each cluster will allow data mining and help initiate functional studies in the rose.

It has been reported that de novo assemblies using RNAseq are highly complex due to allelic and splicing variants and transcriptional noise [58] but also because of sequencing errors and generation of chimeras. Furthermore, read mis-attribution between recently duplicated genes could hamper the discrimination of expression between close paralogs or alleles. This is typically the case for the RcOOMT1 and RcOOMT2 transcripts, which differ only by one SNP in their coding sequence $[6,8]$, and indeed share the same in silico pattern (Additional file 2: Figure S2). Although our in silico data for most analyzed genes were either validated using 
qPCR or coherent with previous published data, gene expression should be validated using independent and sensitive methods, such as $\mathrm{qPCR}$, before functional characterization steps.

\section{Conclusion}

Our de novo sequencing and analyses permitted the generation of information on at least 20997 individual rose peptides, among which are peptides orthologous to at least 14252 different Fragaria proteins. The ROSAseq web portal provides a variety of pre-existing or specifically developed tools and pre-computed searches to conduct in-depth analyses at different levels. The navigation system provided makes it possible to (i) visualize EST cluster characteristics, (ii) explore gene function (iii) analyze gene and protein families (iv) retrieve expression patterns (v) download results of global analyses in tabulated format. The system can be consulted in a variety of ways including via multi-criteria queries based upon annotations, keywords, similarities (using a lucene-based retrieval system) as well as basic similarity searches (BLAST and PatScan) [59,60]. Results are presented with links allowing easy navigation through different sources of information.

The information on Rosa sp gene sequences in this study will also prove extremely useful to generate markers for high density genetic maps and to improve synteny studies with other Rosaceae, in particular Fragaria. Genetic mapping of Rosa has been underway for several years. However, because of the limited information on gene sequences, currently, only about 597 markers have been mapped onto the rose genetic maps, distributed over a length of $530 \mathrm{cM}$ on seven linkage groups [61]. Similarly, synteny studies between Rosa and other Rosaceae such as strawberry has also been hampered because of the limited information on Rosa sp gene sequences [18,62].

Therefore, the ROSAseq database represents a comprehensive resource for transcript detection and accumulation, for genetic mapping and valuable prerequisite to the sequencing of the rose genome.

\section{Methods}

\section{Plant material}

$R$. chinensis $\mathrm{cv}$. 'Old Blush' plants were grown in greenhouse with $16 \mathrm{~h} / 8 \mathrm{~h}$ day/night and $25^{\circ} \mathrm{C} / 14^{\circ} \mathrm{C}$ day/night temperature. For floral transition samples (stage IFL), the terminal parts of growing shoot were harvested and rapidly dissected (removal of young leaves). This stage corresponds to the floral induction and floral initiation stages. Bud bursting samples were collected in the following conditions. Plants were propagated by cuttings and grown until the merging flower bud stage [63]. Bursting axillary buds (DBO) were collected from the upper part of the stem and they swelled with emerging leaf primordia. In contrast, dormant axillary buds (NDB) were harvested from the basal part of the stem and did not exhibit any growth activity. Young flowers (stages 3 to 5) were dissected from developing buds as previously described [29]. Stamens were collected after cytological analysis as previously described [39], different developmental and meiotic stages were pooled to constitute the DET sample. Developmental stages range from early events of organ specification to late ones of anther dehiscence and pollen release.

Young developing leaves and stems were harvested on seven weeks-old Rose plants, multiplied by cuttings and two successive prunings. Roses were cultivated in greenhouse at $24^{\circ} \mathrm{C}$ the day and $18^{\circ} \mathrm{C}$ the night, under a photoperiod of $16 \mathrm{~h}$ light $/ 8 \mathrm{~h}$ dark. The control plants were watered daily for $15 \mathrm{~min}$ by capillarity (FTN), and the stressed plants were submitted to drought by arrest of watering for 4 days (FTS).

\section{RNA preparation}

Total RNA was prepared as previously described [29]. Contaminating DNA was removed using the DNAfreeTM kit (Ambion, Cambridgeshire, UK).

\section{4 and Illumina sequencing}

RNA samples were checked for their integrity on an Agilent 2100 Bioanalyzer (Waldbroon, Germany) according to the manufacturer's instructions. For each sample 25 $\mu \mathrm{g}$ of total RNA was pooled to generate a normalized cDNA library (GATC Biotech) and then used for 454 (Roche) sequencing. Molecular tagging of each of the 13 samples was performed by megaprimer PCR reaction (GATC Biotech) used to generate a tagged - non normalized- 3' cDNA library for Illumina sequencing according to the manufacturers protocols (Illumina).

\section{RNAseq : short reads counting method}

For expression analyses, Illumina reads were mapped on the 80714 rose clusters using the following BLAST parameters. A maximum of 2 mismatches was authorized and only alignments of 24 or more nucleotide were kept. Alignments were filtered keeping only the best score. However, due to dataset complexity some short reads were mapped and affected to more than one cluster. Finally, matches were counted and RPKM computed per cluster and per libraries. Heatmap was generated using RPKM data (ln2 scale) using the $\mathrm{MeV}$ analysis tool [64].

\section{qPCR analysis}

One microgram total RNA (treated with DNase) was used in a reverse transcription assay and qPCR as previously described (Dubois et al., 2011). Expression levels were normalized with Rc-alpha-TUBULIN, RcTCTP, RcACTIN and RcEF1-alpha reference genes [4]. At least 
two independent biological replicates were used for each experiment and two qPCR technical replicates were performed for each biological replicate, with similar results. Primer sequences are available as Additional file 5: Table S3. The correlation between the RNAseq results and qPCR data was assessed by calculating the Pearson's product moment correlation coefficient $[65,66]$.

\section{WEB portal}

ROSAseq Web portal: http://iant.toulouse.inra.fr/R. chinensis

ROSACYC pathway tool portal: http://pathway-tools. toulouse.inra.fr/ROSACYC

\section{Additional files}

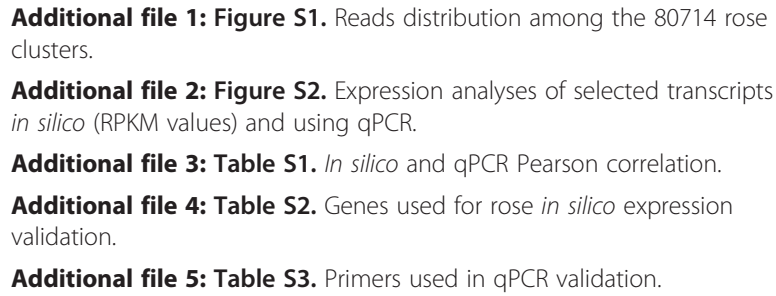

Additional file 3: Table S1. In silico and qPCR Pearson correlation. Additional file 4: Table S2. Genes used for rose in silico expression validation.

Additional file 5: Table S3. Primers used in QPCR validation.

\begin{abstract}
Abbreviations
RAC: White young roots; FTN: Young leaves and stems; FTB: Leaves infected with Botrytis cinerea LR18; FTS: Leaves from water stressed plants; NDB: Dormant axillary buds (vegetative meristem); DBO: Active axillary buds (vegetative meristem); IFL: Floral bud at floral meristem transition; IMO: Floral meristem and early floral organs (sepal, petal, stamens and carpels) development; BFL: Closed flower; OFT: Open flower; DET: Stamens at microsporogenesis and microgametogenesis stages; SEN: Senescent flower; CYN: Rose hip (cynorhodon) from pollination up to early pigmentation; EST: Expressed sequence tag; qPCR: Real time quantitative RT-PCR; SOC1: Supressor of constans1; AG: Agamous; SHP: Shatterproof; SEP: SEPALLATA; MS1: Male sterility1; AMS: Aborted microspore; TDF1: Defective in tapetal development and function 1; RcGDS: GermacreneD synthase; TMB: 1,3,5-trimethoxybenzene; POMT: Phloroglucinol Omethyltransferase; OOMT: Orcinol-O-methyl transferase; DMT: 3,5 dimethoxytoluene; PAAS: Phenylacetaldehyde synthase; PAR: Phenyacetaldehyde reductase.
\end{abstract}

\section{Competing interests}

The authors declare no competing interests.

\section{Authors' contributions}

$A D, S C, O R, B P, L C$ : carried out the molecular genetic studies and participated to the sequence analyses. $M B, A D, O R, J G F F, J-P O, S S, R A, S B$, $M L$ conceived experiments and prepared material. $M B, A D, S C, J G$ : designed and coordinated experiments. SC, LC, JG: analyzed sequence data and designed the ROSAseg and ROSACYC web interface. MB and AD wrote the paper. All authors read and approved the final manuscript.

\section{Acknowledgments}

We thank J. Szecsi and G. Ingram for critical reading of the manuscript. We thank Alexis Lacroix, Yannick Rasmus, Isabelle Desbouchages, Priscilla Angelot (ENS-Lyon), M. Cigna (IMBE, Marseille) and N. Dousset and J. Chameau (INRA Angers) taking care of the plants and P. Vergne, J-C Caissard, P. Jacob, A. Remay, T. Thouroude, N. Leduc, S. Pelleschi-Travier, J. Legourriec et V. Portemer for discussion and help with experiments.

This work was funded by the AIP-Bioressources programme and by the "Biologie Végétale" and the "Génétique et Amélioration des Plantes"
Departments of the French "Institut National de la Recherche Agronomique". This paper is dedicated to our late colleague Jean Paul Onesto.

\section{Author details}

${ }^{1}$ Reproduction et Développement des Plantes UMR INRA-CNRS- Université Lyon 1-ENSL, Ecole Normale Supérieure, 46 allée d'Italie, Lyon Cedex 07 69364, France. ${ }^{2}$ INRA, Laboratoire des Interactions Plantes-Microorganismes (LIPM), UMR441, Castanet-Tolosan F-31326, France. ${ }^{3}$ CNRS, Laboratoire des Interactions Plantes-Microorganismes (LIPM), UMR2594, Castanet-Tolosan F-31326, France. ${ }^{4}$ Laboratoire BVpam, EA2061, Université de Saint-Etienne, Université de Lyon, rue du Dr Michelon, Saint-Etienne F-42023, France. ${ }^{5}$ INRA, Unité de Recherches Intégrées en Horticulture, 400 route des Chappes BP 167, Sophia Antipolis Cedex 06903, France. ${ }^{6}$ Institut de Recherche en Horticulture et Semences (INRA, Agrocacmpus-Ouest, Université d'Angers), SFR 149 QUASAV, Beaucouzé cedex BP 60057-49071, France. Université de Poitiers, UMR CNRS 7267 Écologie et Biologie des Interactions, 40 Av. du Recteur Pineau, Poitiers Cedex 86022, France. ${ }^{8}$ Institut Méditerranéen de Biodiversité et d'Ecologie marine et continentale, UMR Université d'Aix-Marseille- CNRS 7263, Université d'Aix-Marseille, IRD 237, Université d'Avignon, Avenue Escadrille Normandie-NiemenMarseille F-13397, France.

Received: 25 July 2012 Accepted: 6 November 2012

Published: 20 November 2012

\section{References}

1. Channeliere S, Riviere S, Scalliet G, Szecsi J, Jullien F, Dolle C, Vergne P, Dumas $C$, Bendahmane $M$, Hugueney $P$, et al: Analysis of gene expression in rose petals using expressed sequence tags. FEBS Lett 2002, 515(1-3):35-38.

2. Foucher F, Chevalier M, Corre C, Soufflet-Freslon V, Legeai F, Hibrand-Saint Oyant L: New resources for studying the rose flowering process. Genome 2008, 51(10):827-837.

3. Guterman I, Shalit M, Menda N, Piestun D, Dafny-Yelin M, Shalev G, Bar E, Davydov O, Ovadis M, Emanuel M, et al: Rose scent: Genomics approach to discovering novel floral fragrance-related genes. Plant Cell 2002, 14 (10):2325-2338.

4. Dubois A, Remay A, Raymond O, Balzergue S, Chauvet A, Maene M, Pecrix Y, Yang SH, Jeauffre J, Thouroude T, et al: Genomic approach to study floral development genes in Rosa sp. PLoS One 2011, 6(12):e28455.

5. Lavid N, Wang J, Shalit M, Guterman I, Bar E, Beuerle T, Menda N, Shafir S, Zamir D, Adam Z, et al: O-methyltransferases involved in the biosynthesis of volatile phenolic derivatives in rose petals. Plant Physio/ 2002, 129(4):1899-1907.

6. Scalliet G, Journot N, Jullien F, Baudino S, Magnard JL, Channeliere S, Vergne P, Dumas C, Bendahmane M, Cock JM, et al: Biosynthesis of the major scent components 3,5-dimethoxytoluene and 1,3,5trimethoxybenzene by novel rose O-methyltransferases. FEBS Lett 2002, 523(113):118. PII S0014-5793(0002)02956-02953.

7. Scalliet $G$, Lionnet $C$, Le Bechec M, Dutron L, Magnard JL, Baudino S, Bergougnoux V, Jullien F, Chambrier P, Vergne $P$, et al: Role of petalspecific orcinol O-methyltransferases in the evolution of rose scent. Plant Physiol 2006, 140(1):18-29.

8. Scalliet G, Piola F, Douady CJ, Rety S, Raymond O, Baudino S, Bordji K, Bendahmane M, Dumas C, Cock JM, et al: Scent evolution in chinese roses. P Natl Acad Sci USA 2008, 105(15):5927-5932.

9. Shalit M, Guterman I, Volpin H, Bar E, Tamari T, Menda N, Adam Z, Zamir D, Vainstein A, Weiss $D$, et al: Volatile ester formation in roses. Identification of an acetyl-coenzyme A. Geraniol/Citronellol acetyltransferase in developing rose petals. Plant Physiol 2003, 131(4):1868-1876.

10. Guterman I, Masci T, Chen XL, Negre F, Pichersky E, Dudareva N, Weiss D, Vainstein A: Generation of phenylpropanoid pathway-derived volatiles in transgenic plants: Rose alcohol acetyltransferase produces phenylethyl acetate and benzyl acetate in petunia flowers. Plant Mol Biol 2006, 60(4):555-563.

11. Vergne P, Maene M, Gabant G, Chavet A, Debener T, Bendahmane M: Somatic embryogenesis and transformation of the diploid Rosa chinensis cv Old Blush. Plant Cell Tiss Organ Cult 2010, 100:73-81.

12. Krussmann G: The complete book of roses. Portland: Timber Press xii; 1981.

13. Dean R, Van Kan JAL, Pretorius ZA, Hammond-Kosack KE, Di Pietro A, Spanu PD, Rudd JJ, Dickman M, Kahmann R, Ellis J, et al: The Top 10 fungal pathogens in molecular plant pathology. Mol Plant Pathol 2012, 13(4):414-430. no-no. 
14. Vrind TA: The Botrytis problem in figures. In: Proceedings of the VIIIth International Symposium on Postharvest Physiology of Ornamental Plants. Edited by Marissen NWWGV; 2005:99-102.

15. Hernandez D, Francois P, Farinelli L, Osteras M, Schrenzel J: De novo bacterial genome sequencing: millions of very short reads assembled on a desktop computer. Genome Res 2008, 18(5):802-809.

16. Pertea G, Huang X, Liang F, Antonescu V, Sultana R, Karamycheva S, Lee Y, White J, Cheung F, Parvizi B, et al: TIGR Gene Indices clustering tools (TGICL): a software system for fast clustering of large EST datasets. Bioinformatics 2003, 19(5):651-652.

17. Krizek BA, Fletcher JC: Molecular mechanisms of flower development: an armchair guide. Nat Rev Genet 2005, 6(9):688-698.

18. Gar O, Sargent DJ, Tsai CJ, Pleban T, Shalev G, Byrne DH, Zamir D: An autotetraploid linkage map of rose (Rosa hybrida) validated using the strawberry (Fragaria vesca) genome sequence. PLoS One 2011, 6(5):e20463.

19. Jung S, Cestaro A, Troggio M, Main D, Zheng P, Cho I, Folta KM, Sosinski B, Abbott A, Celton JM, et al: Whole genome comparisons of fragaria, prunus and malus reveal different modes of evolution between rosaceous subfamilies. BMC Genomics 2012, 13:129.

20. Shulaev V, Sargent DJ, Crowhurst RN, Mockler TC, Folkerts O, Delcher AL, Jaiswal P, Mockaitis K, Liston A, Mane SP, et al: The genome of woodland strawberry (Fragaria vesca). Nat Genet 2011, 43(2):109-116.

21. Gouzy J, Carrere S, Schiex T: FrameDP: sensitive peptide detection on noisy matured sequences. Bioinformatics 2009, 25(5):670-671.

22. Quevillon E, Silventoinen V, Pillai S, Harte N, Mulder N, Apweiler R, Lopez R: InterProScan: protein domains identifier. Nucleic Acids Res 2005, 33(Web Server issue):W116-W120.

23. Li L, Stoeckert CJ, Roos DS: OrthoMCL: Identification of ortholog groups for eukaryotic genomes. Genome Res 2003, 13(9):2178-2189.

24. Karp PD, Paley SM, Krummenacker M, Latendresse M, Dale JM, Lee TJ, Kaipa P, Gilham F, Spaulding A, Popescu L, et al: Pathway tools version 13.0: integrated software for pathway/genome informatics and systems biology. Brief Bioinform 2010, 11(1):40-79.

25. Mortazavi A, Williams BA, McCue K, Schaeffer L, Wold B: Mapping and quantifying mammalian transcriptomes by RNA-Seq. Nat Methods 2008, 5(7):621-628.

26. Baker M: De novo genome assembly: what every biologist should know pp 333-337. Nat Methods 2012, 9:333-337.

27. Remay A, Lalanne D, Thouroude T, Le Couviour F, Hibrand-Saint Oyant L, Foucher F: A survey of flowering genes reveals the role of gibberellins in floral control in rose. Theor Appl Genet 2009, 119(5):767-781.

28. Hibino $Y$, Kitahara $K$, Hirai S, Matsumoto S: Structural and functional analysis of rose class B MADS-box genes 'MASAKO BP, euB3, and B3': Paleo-type AP3 homologue 'MASAKO B3' association with petal development. Plant Sci 2006, 170:778-785.

29. Dubois A, Raymond O, Maene M, Baudino S, Langlade NB, Boltz V, Vergne $P$, Bendahmane M: Tinkering with the C-function: a molecular frame for the selection of double flowers in cultivated roses. PLoS One 2010, 5(2):e9288.

30. Kitahara K, Hibino Y, Aida R, Matsumoto S: Ectopic expression of the rose AGAMOUS-like MADS-box genes 'MASAKO C1 and D1' causes similar homeotic transformation of sepal and petal in Arabidopsis and sepal in Torenia. Plant Sci 2004, 166(5):1245-1252.

31. Causier B, Castillo R, Zhou J, Ingram R, Xue Y, Schwarz-Sommer Z, Davies B: Evolution in action: following function in duplicated floral homeotic genes. Current biology: CB 2005, 15(16):1508-1512.

32. Seymour GB, Ryder CD, Cevik V, Hammond JP, Popovich A, King GJ, Vrebalov J, Giovannoni JJ, Manning K: A SEPALLATA gene is involved in the development and ripening of strawberry (FragariaXananassa Duch.) fruit, a non-climacteric tissue. J Exp Bot 2011, 62(3):1179-1188.

33. Vrebalov J, Pan IL, Arroyo AJM, McQuinn R, Chung M, Poole M, Rose JKC, Seymour G, Grandillo S, Giovannoni J, et al: Fleshy fruit expansion and ripening Are regulated by the tomato SHATTERPROOF gene TAGL1. Plant Cell 2009, 21(10):3041-3062.

34. Ariizumi T, Toriyama K: Genetic regulation of sporopollenin synthesis and pollen exine development. Annu Rev Plant Biol 2011, 62:437-460.

35. Borg M, Brownfield L, Twell D: Male gametophyte development: a molecular perspective. J Exp Bot 2009, 60(5):1465-1478.

36. Chang F, Wang $\mathrm{Y}$, Wang $\mathrm{S}$, Ma H: Molecular control of microsporogenesis in Arabidopsis. Curr Opin Plant Biol 2011, 14(1):66-73.

37. $\mathrm{Ma} \mathrm{H}$ : A molecular portrait of Arabidopsis meiosis. The Arabidopsis book Am Soc Plant Biologists 2006, 4:e0095.
38. Mercier R, Grelon M: Meiosis in plants: ten years of gene discovery. Cytogenet Genome Res 2008, 120(3-4):281-290.

39. Pecrix Y, Rallo G, Folzer H, Cigna M, Gudin S, Le Bris M: Polyploidization mechanisms: temperature environment can induce diploid gamete formation in Rosa sp. J Exp Bot 2011, 62(10):3587-3597.

40. Wilson ZA, Zhang DB: From Arabidopsis to rice: pathways in pollen development. J Exp Bot 2009, 60(5):1479-1492.

41. Sorensen AM, Krober S, Unte US, Huijser P, Dekker K, Saedler H: The Arabidopsis ABORTED MICROSPORES (AMS) gene encodes a MYC class transcription factor. Plant J 2003, 33(2):413-423.

42. Wilson ZA, Morroll SM, Dawson J, Swarup R, Tighe PJ: The Arabidopsis MALE STERILITY1 (MS1) gene is a transcriptional regulator of male gametogenesis, with homology to the PHD-finger family of transcription factors. Plant J 2001, 28(1):27-39.

43. Yang C, Vizcay-Barrena G, Conner K, Wilson ZA: MALE STERILITY1 is required for tapetal development and pollen wall biosynthesis. Plant Cell 2007, 19(11):3530-3548.

44. Zhu J, Chen H, Li H, Gao JF, Jiang H, Wang C, Guan YF, Yang ZN: Defective in Tapetal development and function 1 is essential for anther development and tapetal function for microspore maturation in Arabidopsis. Plant J 2008, 55(2):266-277.

45. Hird DL, Worrall D, Hodge R, Smartt S, Paul W, Scott R: The anther-specific protein encoded by the Brassica napus and Arabidopsis thaliana A6 gene displays similarity to beta-1,3-glucanases. Plant J 1993, 4(6):1023-1033.

46. Kaur J, Sebastian J, Siddiqi I: The Arabidopsis-mei2-like genes play a role in meiosis and vegetative growth in Arabidopsis. Plant Cell 2006, 18(3):545-559.

47. Yang M, Hu Y, Lodhi M, McCombie WR, Ma H: The Arabidopsis SKP1-LIKE1 gene is essential for male meiosis and may control homologue separation. Proc Natl Acad Sci USA 1999, 96(20):11416-11421.

48. Porat R, Lu P, O'Neill SD: Arabidopsis SKP1, a homologue of a cell cycle regulator gene, is predominantly expressed in meristematic cells. Planta 1998, 204(3):345-351.

49. de Azevedo Souza C, Kim SS, Koch S, Kienow L, Schneider K, McKim SM, Haughn GW, Kombrink E, Douglas CJ: A novel fatty acyl-CoA synthetase is required for pollen development and sporopollenin biosynthesis in arabidopsis. Plant Cell 2009, 21(2):507-525.

50. Matsuno M, Compagnon V, Schoch GA, Schmitt M, Debayle D, Bassard JE, Pollet B, Hehn A, Heintz D, Ullmann P, et al: Evolution of a novel phenolic pathway for pollen development. Science 2009, 325(5948):1688-1692.

51. Kim HJ, Oh SA, Brownfield L, Hong SH, Ryu H, Hwang I, Twell D, Nam HG: Control of plant germline proliferation by SCF(FBL17) degradation of cell cycle inhibitors. Nature 2008, 455(7216):1134-1137.

52. Wu S, Watanabe N, Mita S, Dohra H, Ueda Y, Shibuya M, Ebizuka Y: The key role of phloroglucinol O-methyltransferase in the biosynthesis of Rosa chinensis volatile 1,3,5-trimethoxybenzene. Plant Physiol 2004, 135(1):95-102

53. Huang FC, Horvath G, Molnar P, Turcsi E, Deli J, Schrader J, Sandmann G, Schmidt H, Schwab W: Substrate promiscuity of RdCCD1, a carotenoid cleavage oxygenase from Rosa damascena. Phytochemistry 2009, 70(4):457-464

54. Huang FC, Molnar P, Schwab W: Cloning and functional characterization of carotenoid cleavage dioxygenase 4 genes. J Exp Bot 2009, 60(11):3011-3022.

55. Kaminaga Y, Schnepp J, Peel G, Kish CM, Ben-Nissan G, Weiss D, Orlova I, Lavie O, Rhodes D, Wood K, et al: Plant phenylacetaldehyde synthase is a bifunctional homotetrameric enzyme that catalyzes phenylalanine decarboxylation and oxidation. J Biol Chem 2006, 281(33):23357-23366.

56. Sakai M, Hirata H, Sayama H, Sekiguchi K, Itano H, Asai T, Dohra H, Hara M, Watanabe N: Production of 2-phenylethanol in roses as the dominant floral scent compound from L-phenylalanine by two key enzymes, a PLP-dependent decarboxylase and a phenylacetaldehyde reductase. Biosci Biotechnol Biochem 2007, 71(10):2408-2419.

57. Chen XM, Kobayashi H, Sakai M, Hirata H, Asai T, Ohnishi T, Baldermann S, Watanabe N: Functional characterization of rose phenylacetaldehyde reductase (PAR), an enzyme involved in the biosynthesis of the scent compound 2-phenylethanol. J Plant Physiol 2011, 168(2):88-95.

58. Pickrell JK, Pai AA, Gilad Y, Pritchard JK: Noisy splicing drives mRNA isoform diversity in human cells. PLoS Genet 2010, 6(12):e1001236.

59. Altschul SF, Madden TL, Schaffer AA, Zhang J, Zhang Z, Miller W, Lipman DJ: Gapped BLAST and PSI-BLAST: a new generation of protein database search programs. Nucleic Acids Res 1997, 25(17):3389-3402. 
60. Dsouza M, Larsen N, Overbeek R: Searching for patterns in genomic data. Trends in genetics: TIG 1997, 13(12):497-498.

61. Spiller M, Linde M, Hibrand-Saint Oyant L, Tsai CJ, Byrne DH, Smulders MJ, Foucher F, Debener T: Towards a unified genetic map for diploid roses. Theor Appl Genet 2011, 122(3):489-500.

62. I wata $\mathrm{H}$, Gaston A, Remay A, Thouroude T, Jeauffre J, Kawamura K, Oyant LH, Araki T, Denoyes B, Foucher F: The TFL1 homologue KSN is a regulator of continuous flowering in rose and strawberry. Plant J 2012, 69(1):116-125.

63. Girault T, Abidi F, Sigogne M, Pelleschi-Travier S, Boumaza R, Sakr S, Leduc $\mathrm{N}$ : Sugars are under light control during bud burst in Rosa sp. Plant Cell Environ 2010, 33(8):1339-1350.

64. Saeed Al, Bhagabati NK, Braisted JC, Liang W, Sharov V, Howe EA, Li J, Thiagarajan M, White JA, Quackenbush J: TM4 microarray software suite. Methods Enzymol 2006, 411:134-193.

65. Coppack SW: Limitations of the Pearson product-moment correlation. Clin Sci (Lond) 1990, 79(3):287.

66. Liu Y, Meng $Q$, Chen R, Wang J, Jiang S, Hu Y: A new method to evaluate the similarity of chromatographic fingerprints: weighted pearson productmoment correlation coefficient. J Chromatogr Sci 2004, 42(10):545-550.

doi:10.1186/1471-2164-13-638

Cite this article as: Dubois et al.: Transcriptome database resource and gene expression atlas for the rose. BMC Genomics 2012 13:638.

\section{Submit your next manuscript to BioMed Central and take full advantage of:}

- Convenient online submission

- Thorough peer review

- No space constraints or color figure charges

- Immediate publication on acceptance

- Inclusion in PubMed, CAS, Scopus and Google Scholar

- Research which is freely available for redistribution 